\title{
Harvested Energy Maximization of SWIPT System with Popularity Cache Scheme in Dense Small Cell Networks
}

\author{
Xuefei Peng and Jiandong $\mathrm{Li}$ (i) \\ The State Key Laboratory of Integrated Service Networks, Xidian University, Xian 710071, China \\ Correspondence should be addressed to Jiandong Li; jdli@xidian.edu.cn
}

Received 10 January 2019; Revised 7 March 2019; Accepted 27 March 2019; Published 1 July 2019

Guest Editor: Zoran Stamenkovic

Copyright (C) 2019 Xuefei Peng and Jiandong Li. This is an open access article distributed under the Creative Commons Attribution License, which permits unrestricted use, distribution, and reproduction in any medium, provided the original work is properly cited.

\begin{abstract}
In this paper, we propose a harvested energy maximization problem of simultaneous wireless information and power transfer (SWIPT) system with popularity cache scheme in dense small cell networks. Firstly, network model, content request, and popularity cache schemes are provided in the system model. Then, we establish a harvested energy maximization problem of SWIPT system with popularity cache scheme in dense small cell networks, where maximum transmit power of small cell base stations (SBSs), minimum rate requirement, i.e., quality of service (QoS) of user terminals (UTs), and power splitting ratio are considered. Further, an iterative power splitting ratio and power allocation optimization (IPSPA) algorithm is proposed to solve the formulated problem. Finally, the better performance of our proposed method is demonstrated through a number of simulations. These results are of significance for maximizing harvesting energy of UTs and reducing consumption of backhaul resources and energy.
\end{abstract}

\section{Introduction}

In future, the fifth generation (5G) wireless network will be characterized by energy-limited environment that is inconvenient to connect with fixed charging facilities [1-3]. Energy storage can provide power for UTs by storing energy in their batteries. Traditional energy harvesting method cannot achieve information transmission when it stores energy. Compared with traditional energy storage methods, simultaneous wireless information and power transfer (SWIPT) is a promising energy harvesting technology that allows UTs to harvest energy from radio signals by sensing radio frequency sent by surrounding small cell base stations (SBSs). Moreover, SWIPT system can guarantee UTs performing information decoding (ID) and energy harvesting (EH) simultaneously without interrupting data transmission through adopting power splitters. SWIPT is a key technical innovation that promotes the development of the energy field and may bring vital changes to wireless communication networks [13]. Nevertheless, dense small cell deployment in 5G wireless communication networks will introduce several major technical challenges. On the one hand, spectrum sharing among small cells leads to severe interference in the networks.
On the other hand, this large deployment creates a new burden on backhaul links for all the SBSs. Therefore, it is necessary to maximize harvested energy of SWIPT system by exploring interference and increasing the time for energy harvesting, as well as save backhaul resources and energy through reducing backhaul load. In this paper, we explore both useful and interference radio frequency signals in dense small cell networks as the energy harvesting source and introduce SBSs with the ability of caching in SWIPT system to decrease the distance between UTs and their desired contents. Here, note that contents in this paper refer to voice, video, data or files, etc. Aiming at maximizing harvested energy of the SWIPT system with popularity cache scheme and reducing backhaul load, we propose a harvested energy maximization problem considering constraints of the transmit power of SBSs, quality of service (QoS) of UTs, and power splitting ratio of SWIPT system. We firstly determine content request and popularity cache schemes. Then, we provide the popularity cache-based user association scheme and resource allocation scheme. Further, we propose an iterative power splitting ratio and power allocation optimization (IPSPA) algorithm to solve the formulated problem. Our proposed scheme has two advantages compared to traditional SWIPT 
system in which SBSs do not have the capability of caching from local SBSs and have to fetch each content (voice, video, data or a file, etc.) from the core network through backhaul links. One advantage is reduction of the time of fetching contents from core network by obtaining some of the contents from local SBSs, thereby providing more time to harvest energy and improve energy harvesting efficiency. The other advantage is the reduction of backhaul links load through employing the proposed popularity caching scheme of SBSs.

The paper is organized as follows. The description of related work is given in Section 2. In Section 3, we outline the system model including network model, content request, and popularity cache schemes. In Section 4, we formulate and solve the harvested energy maximization problem of SWIPT system with popularity cache scheme in dense small cell networks. Complexity analysis of the proposed solution is given in Section 5. In Section 6, a number of simulations are provided. Finally, Section 7 concludes the paper.

\section{Related Work}

Recently, some works have studied energy problem about SWIPT system from the aspects of optimizing time, power, power splitting ratio, and channel resource [4-12]. The coupled time and power allocation problem of user-centric energy efficiency maximization problem considering time switching protocol for wireless powered communication network was solved by the proposed iterative resource allocation scheme in [4]. In [5], the authors presented optimal parameter design for the harvested energy maximization problem with the constraint of a minimum information rate requirement for SWIPT system, which included both channel assignment and power allocation. Reference [6] aimed to maximize the sum harvested energy considering constraints of rate, transmit power, and subchannel assignment firstly. Then, a scheme for harvested energy maximization of worst case individual link was proposed to ensure fairness among different links. In [7, 8], the authors studied SWIPT system in fog-computing networks. In [7], authors aimed to minimize energy cost of ultralow power devices through optimizing time slots and power allocation. Moreover, the conclusion that harvested energy would decrease when the number of computing operations became larger and the distance between access point and ultralow power devices increased was obtained in [7]. In [8], a quantum particle swarm optimization algorithm was introduced to minimize the total energy consumption at the source node through a power, time, and data allocation scheme while the latency requirement was maintained. Reference [9] considered a decode-and-forward relaying network based on SWIPT, where the optimal dynamic power splitting ratio and time allocation ratio for minimizing the outage probability were obtained through split-step iterative method. Moreover, the problem of maximizing the instantaneous channel capacity was solved by an alternate convex optimization method. A dynamic algorithm with a control parameter to jointly allocate the transmission power and time switching factor was proposed in [10]. Authors in [11] proposed a unified framework to optimize the influence of SWIPT on the system throughput and energy efficiency through both time splitting and power splitting schemes. In [12], the authors established an iterative suboptimal resource allocation algorithm for multiuser to minimize total transmit power of the network. Besides, authors in $[13,14]$ derived exact closedform expressions for the outage probability to evaluate the impact of power allocation on outage probability. However, SWIPT system with caching capability is not considered in [4-14].

Additionally, a number of literature works have concentrated on caching problems [15-21]. Authors in [15] employed physical caching scheme that brought both cacheinduced cooperation and the cache-assisted multihopping gains. In [16], the authors considered density of SBSs, memory, and backhaul optimization problem considering wireless QoS constraint, where most popular contents prior caching scheme were adopted. In [17], the authors proposed a resource allocation optimization problem to minimize the blocking probability considering caching capacity and backhaul limitation, where multidimensional Markov chains were employed to evaluate the performance of network. Authors in [18] discussed cooperative random and uniform caching scheme between base stations (BSs) and devices to maximize the successful transmission probability, and the equivalently transformed biconvex problem was solved by block coordinate descent algorithm. In [19], the authors analyzed the probability that mobile users could successfully download their requested files from the SBSs based on partitioned file groups, random and uniform caching strategy, where impact of LoS and NLoS, transmit power, and density of SBSs were considered. In [20], the authors proposed three algorithms of power allocation to achieve different objectives, i.e., sum transmission rate maximization, average outage probability minimization, and average satisfaction maximization, to promote the performance of cache-aided small cell networks with limited backhaul capacity, where a fix ratio of requested contents was assumed to exist in local caches of SBSs, and other contents should be fetched from core networks. In [21], the authors proposed a power control method for energy harvesting SBSs and revealed that the energy and sum-throughput performances of these SBSs were influenced by energy harvesting and caching abilities. However, energy harvesting of SWIPT system and caching of contents are not considered simultaneously in [1521]. follows:

The main contributions of this paper are summarized as

(i) Our proposed SWIPT system with popularity cache scheme has the advantages of improving harvested energy of UTs, saving backhaul resources and energy.

(ii) We formulate the harvested energy maximization problem of SWIPT system in dense small cell networks considering the maximum transmit power of SBSs, minimum rate requirement, i.e., QoS of UTs, and power splitting ratio. 


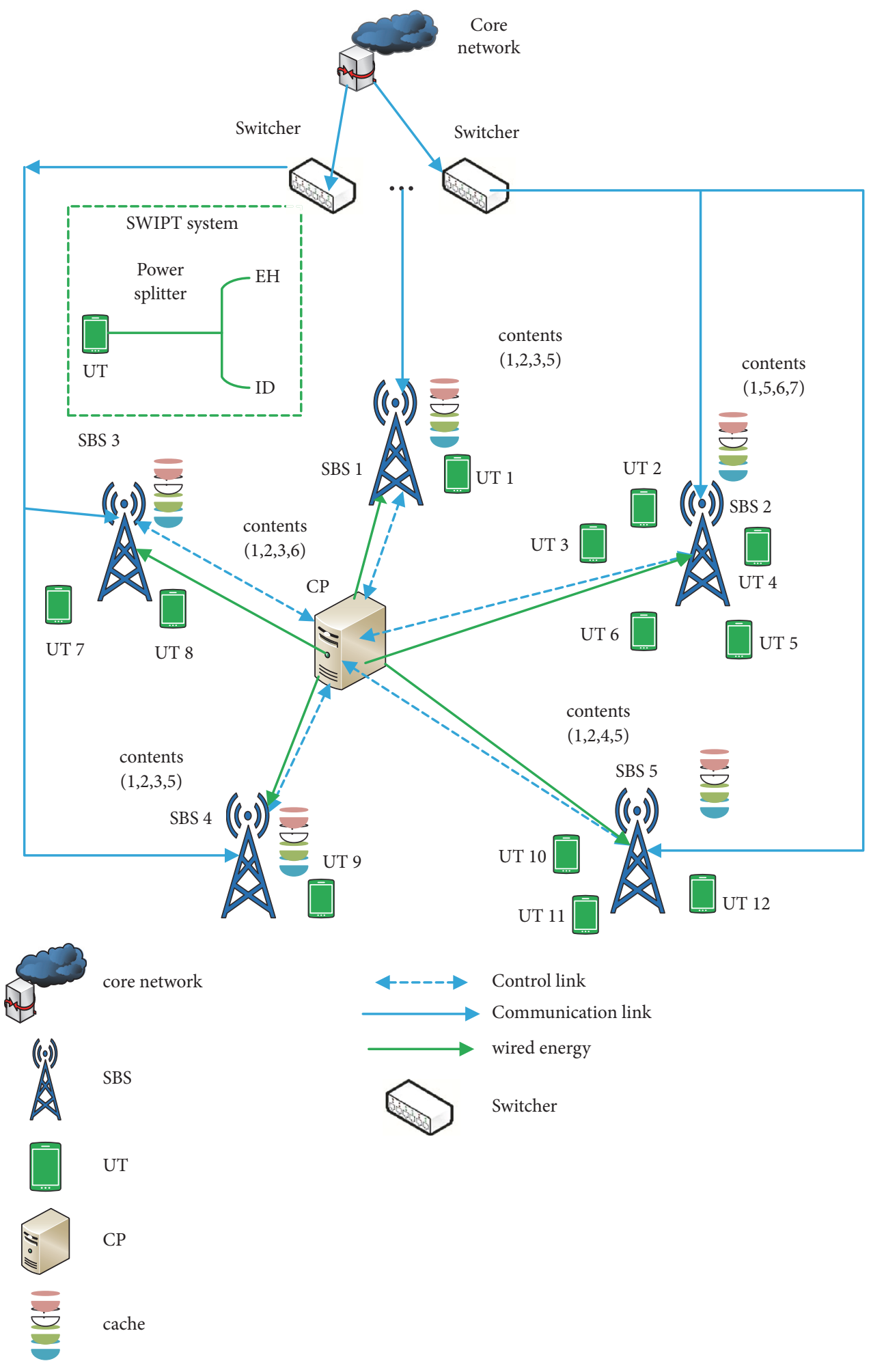

FIGURE 1: Network model.

(iii) An IPSPA algorithm is proposed to solve the formulated harvested energy maximization problem.

(iv) The influence of different parameters on the network performance is evaluated in the simulation.

\section{System Model}

3.1. Network Model. As shown in Figure 1, we consider a SWIPT system with popularity cache scheme in dense small networks. All UTs are uniformly and randomly distributed 
TABLE 1: A simplified example of popularity cache-based user association scheme.

\begin{tabular}{lcc}
\hline Requested content & whether requested content cached in the nearest SBS & Association scheme \\
\hline content1 (UT1) & Yes (SBS1) & case1 \\
content2 (UT2) & No (SBS2) & case2 \\
content3 (UT3) & No (SBS2) & case1 \\
content6 (UT4) & Yes (SBS2) & case1 \\
content1 (UT5) & Yes (SBS2) & case1 \\
content7 (UT6) & Yes (SBS2) & case1 \\
content3 (UT7) & Yes (SBS3) & case1 \\
content6 (UT8) & Yes (SBS3) & case1 \\
content2 (UT9) & Yes (SBS4) & case1 \\
content4 (UT10) & Yes (SBS5) & case1 \\
content5 (UT11) & Yes (SBS5) & case2 \\
content7 (UT12) & No (SBS5) & \\
\hline
\end{tabular}

in the coverage range of SBSs, and all SBSs are deployed in the same spectrum with the bandwidth $\mathrm{BW}$ and $\mathrm{N}_{\mathrm{BW}}$ orthogonal frequency division multiple access (OFDMA) resource blocks (RBs). Let $\mathcal{S}=\{1,2, \ldots, j, \ldots, \mathrm{S}\}$ and $\mathscr{K}=\{1,2, \ldots, i, \ldots \mathrm{K}\}$ denote the set of SBSs and UTs, respectively. Moreover, we let $\mathbf{x}=\left\{x_{1}, x_{2}, \ldots, x_{j}, \ldots, x_{\mathrm{K}}\right\}$ denote information symbols sent by corresponding SBSs 1 to $\mathrm{S}$, where $\mathrm{E}\left(x_{j}{ }^{2}\right)=1$ is the energy of symbol $x_{j}$. A central processor $(\mathrm{CP})$ is responsible for providing energy to the SBSs that provide service to their associated UTs, and switches are used to connect the core network and SBSs. Optical fiber is used as the medium of backhaul links. Moreover, a power splitter is configured in each user terminal (UT) forming a SWIPT system to sense the radio frequency energy of the surrounding SBSs and split the received signal into two parts, which are used for ID and EH.

3.2. Content Request and Popularity Cache Schemes. According to $[17,18]$, the request probability mass function of all contents can be modeled as a Zipf distribution. The probability of the $f$-ranked popularity content requested by UT $i$ can be expressed as follows:

$$
p p_{i, f}=\frac{1 / f^{\alpha}}{\sum_{f=1}^{\mathrm{F}} 1 / f^{\alpha}} \quad(f \in \mathscr{F})
$$

where $\alpha$ is the parameter of the model reflecting the popularity distribution of content $f$. The larger $\alpha$ represents the fewer popular contents undertaking the majority of the requests.

For the contents caching problem, we assume that $F$ popular contents with the same size constitute a content library, which can be denoted as $\mathscr{F}=\{1,2, \ldots, f, \ldots \mathrm{F}\}$. Let C denote the cache size of a small cell base station (SBS). Different from current caching models [16-20], we assume that all contents are precached in each cache space of an SBS according to Zipf distribution, and the cache spaces of an SBS are independent of each other. Therefore, the contents cached in all the spaces of SBS $j$ obey binomial distribution $\mathrm{B}\left(\mathrm{C}, p p_{i, j, f}\right)$, where $\mathrm{C}$ is the cache size of SBS $j$ and $p p_{i, j, f}=$ $p p_{i, f}$ is the probability that a cache space of SBS $j$ caches content $f$ that user $i$ requests.

\section{Problem Formulation and Solution}

We study the harvested energy maximization problem of SWIPT system with popularity cache scheme through three processes, which are popularity cache-based user association scheme, resource allocation, and iterative power splitting ratio and power allocation optimization.

4.1. Popularity Cache-Based User Association Scheme. There are two cases for the popularity cache-based user association scheme. Case1: a number of UTs can obtain the required contents from their nearest local SBSs that cache the required contents without consuming backhaul resources. Case2: some UTs cannot access the contents directly from their nearest (minimum path loss) SBSs. Therefore, these UTs will fetch the required contents from the core network and choose the SBSs nearest them as their serving SBSs. We would like to use a simplified example of a snapshot shown in Figure 1 to illustrate the proposed user association scheme. In Table 1, the required contents of UT1, UT4, UT5, UT6, UT7, UT8, UT9, UT10, and UT11 are cached in their nearest SBSs, so they can obtain their required contents from local SBSs. However, the required contents of UT2, UT3, and UT12 are not cached in their nearest SBSs; they have to fetch their required contents from the core network and then be served by their nearest SBSs. Let $\mathscr{B}_{i}$ denote the set of SBS that UT $i$ is associated with, which is determined by the proposed popularity cache-based user association scheme and can be expressed as follows.

$$
B_{i}=\left\{\begin{array}{l}
\text { local nearest SBS (case1) } \\
\text { nearest SBS that fetched contents by backhaul link (case2) }
\end{array}\right.
$$


Then, we can derive the probability that at least one cache space of SBS $j$ caches content $f$ that $\mathrm{UT} i$ requests as follows:

$$
p p_{i, j, f}^{\mathrm{A}}=1-\left(1-p p_{i, j, f}\right)^{\mathrm{C}} .
$$

Further, we can derive the association probability that UT $i$ associated with its nearest SBS belongs to $B_{i}$ when it requests content $f$, i.e., hit ratio, as follows:

$$
\begin{aligned}
\mathrm{A}_{i, j, f}^{h i t}=p p_{i, f} \times\left(p p_{i, j, f}^{\mathrm{A}}\right)^{\left|B_{i}\right|} & \\
& \forall j \in \mathcal{S}, i \in \mathscr{K} \text {, and } f \in \mathscr{F} .
\end{aligned}
$$

Therefore, we can derive the probability that UT $i$ obtains content $f$ from local SBS and backhaul link as follows:

$$
\zeta_{i, j, f}= \begin{cases}\mathrm{A}_{i, j, f}^{h i t}, & \text { local nearest SBS (case1) } \\ 1-\mathrm{A}_{i, j, f}^{\text {hit }}, & \text { backhaul link (case } 2) .\end{cases}
$$

Depending on practical network measurement described in $[22,23]$, UTs fetch contents through backhaul link will introduce delay. We denote by $\eta_{B H}$ the energy harvesting impact factor, which represents energy harvesting efficiency affected backhaul link. Here, $\eta_{B H}=1 / m$ and $\mathrm{T}_{B H}=m \mathrm{~T}$, where $\mathrm{T}_{B H}$ is the time of UTs obtaining contents from the core network, $\mathrm{T}$ is the time of UTs obtaining contents from local SBSs caching required contents, and $m>1$ is the energy harvesting delay impact factor that denotes the relationship between the time of UTs obtaining desired contents from backhaul link and local SBSs. The illustration of energy harvesting delay impact factor $m$ is shown in Figure 2. We set $\eta_{B H}$ and $m$ on the basis of $[22,23]$ in our simulation.

Consequently, we can derive the energy harvesting coefficient $\ell_{i, j, f}$ as follows:

$$
\begin{aligned}
& \ell_{i, j, f} \\
& \quad= \begin{cases}\mathrm{A}_{i, j, f}^{\text {hit }}, & \text { local nearest SBS (casel) } \\
\left(1-\mathrm{A}_{i, j, f}^{\text {hit }}\right) \times \eta_{B H}, & \text { backhaul link (case } 2),\end{cases}
\end{aligned}
$$

where energy harvesting coefficient $\ell_{i, j, f}$ represents energy harvesting efficiency of UTs obtaining contents from local SBSs (case 1) and backhaul link (case 2), and energy harvesting impact factor $\eta_{B H}$ indicates the energy harvesting efficiency affected by backhaul link.

4.2. Resource Allocation. After the aforementioned analysis of popularity cache-based user association scheme, we will determine the RBs assigned to each UT. Assume that $\mathrm{N}_{\mathrm{BW}}$ OFDMA RBs of the system are equally allocated to the UTs associated with each SBS. Each RB has the bandwidth of $0.18 \mathrm{MHz}$. Therefore, there is no intracell interference, while there exists intercell interference since all the RBs of the system are shared among different SBSs. Let $\mathcal{N}=\{1,2, \ldots, n, \ldots \mathrm{N}\}$ denote the set of subbands allocated to the UTs deployed in the network, where the

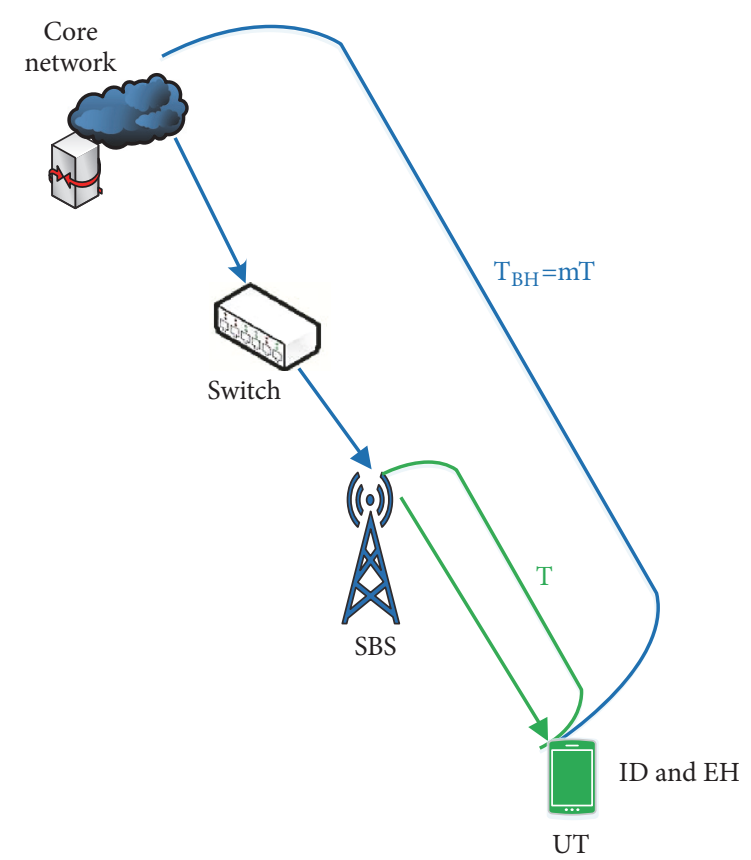

FIGURE 2: Illustration of energy harvesting delay impact factor $m$.

resource blocks allocated to a UT are defined as a subband. Besides, let $\mathscr{K}_{j}$ denote the set of UTs that SBS $j$ associated with. Then, the subbandwidth of UT $i$ associated with SBS $j$ can be denoted as $\mathrm{BW}_{i}=0.18 \times\left[\mathrm{N}_{\mathrm{BW}} /\left|\mathscr{K}_{j}\right|\right] \mathrm{MHz}$, where $\left[\mathrm{N}_{\mathrm{BW}} /\left|\mathscr{K}_{j}\right|\right]$ represents the maximum integer not larger than $\left[\mathrm{N}_{\mathrm{BW}} /\left|\mathscr{K}_{j}\right|\right]$.

4.3. Iterative Power Splitting Ratio and Power Allocation Optimization. After the analytical processes of popularity cachebased user association and resource allocation schemes, we can determine the set of SBS that each UT is associated with, energy harvesting coefficient of each content requested by UTs, and the subbandwidths allocated to the UTs. In this phase, we maximize the harvested energy of the SWIPT system considering the minimum rate requirement, i.e., QoS of UTs, by optimizing power splitting ratio and power allocation from SBSs to UTs. Let $\mathbf{p}=\left(p_{i, j, n}\right)_{\mathrm{K} \times \mathrm{S} \times \mathrm{N}}$ denote the transmit power of SBSs, where $p_{i, j, n}$ represents the transmit power from SBS $j$ to UT $i$ on subband $n$. Then, the received signal of UT $i$ on subband $n$ from SBS $j$ can be written as

$$
y_{i}=\sqrt{p_{i, j, n}} h_{i, j, n} x_{j}+\sum_{j \notin \mathscr{B}_{i}} \sqrt{p_{i, j, n}} h_{i, j, n} x_{j}+n_{i},
$$

where $x_{j} \in \mathbf{x}$ and $n_{i} \sim \mathscr{C} \mathcal{N}\left(0, \sigma^{2}\right)$. We specify $\rho$ as the power splitting ratio, which denotes the ratio of signal strength that the ID module occupied in the total received signal. Then, we can obtain the received signal of UT $i$ for ID module as

$$
\begin{aligned}
y_{i}^{\mathrm{ID}}= & \sqrt{\rho}\left(\sqrt{p_{i, j, n}} h_{i, j, n} x_{j}+\sum_{j \notin \mathscr{B}_{i}} \sqrt{p_{i, j, n}} h_{i, j, n} x_{j}+n_{i}\right) \\
& +n_{i}^{\mathrm{ID}},
\end{aligned}
$$


and the signal of UT $i$ for energy harvesting as

$$
\begin{aligned}
& y_{i}^{\mathrm{EH}} \\
& \quad=\sqrt{1-\rho}\left(\sqrt{p_{i, j, n}} h_{i, j, n} x_{j}+\sum_{j \notin \mathscr{B}_{i}} \sqrt{p_{i, j, n}} h_{i, j, n} x_{j}+n_{i}\right),
\end{aligned}
$$

respectively, where $n_{i}^{\mathrm{ID}} \sim \mathscr{C} \mathscr{N}\left(0,\left(\sigma_{i}^{\mathrm{ID}}\right)^{2}\right)$ is the additive white Gaussian noise introduced by the ID module. It can be observed that the signals used to harvest energy come from the serving SBS and the interfering SBSs. We can derive the achievable SINR of UT $i$ when it requests content $f$ as follows:

$$
\operatorname{SINR}_{i, f}=\frac{\rho\left|\sqrt{p_{i, j, n}} h_{i, j, n}\right|^{2}}{\rho \sum_{j \notin \mathscr{B}_{i}}\left|\sqrt{p_{i, j, n}} h_{i, j, n}\right|^{2}+\rho \sigma^{2}+\left(\sigma_{i}^{\mathrm{ID}}\right)^{2}} .
$$

Since the power of $n_{i}$ is too low, so we can obtain the average harvested energy of UT $i$ when it requests content $f$ from SBS $j$ on subband $n$ by ignoring the power of noise $n_{i}$ as follows:

$$
\begin{aligned}
\mathrm{EH}_{i, f} & \\
= & (1-\rho) \\
& \times \ell_{i, j, f}\left(\left|\sqrt{p_{i, j, n}} h_{i, j, n}\right|^{2}+\sum_{j \notin \mathscr{B}_{i}}\left|\sqrt{p_{i, j, n}} h_{i, j, n}\right|^{2}\right),
\end{aligned}
$$

where we assume that the EH module has the perfect energy conversion efficiency 1 . Further, we can obtain the rate of UT $i$ when it requests content $f$ as follows:

$$
\mathrm{R}_{i, f}=\mathrm{BW}_{i} \times \log _{2}\left(1+\operatorname{SINR}_{i, f}\right),
$$

where $\mathrm{BW}_{i}$ is the bandwidth of UT $i$ determined in the process of resource allocation. Eventually, we can construct harvested energy maximization problem of the network as follows:

$$
\begin{array}{ll}
\max _{(\rho, \mathbf{p})} & \sum_{i \in \mathscr{K}, f \in \mathscr{F}} \mathrm{EH}_{i, f} \\
\text { s.t. } & (13 \mathrm{a}) \quad 0 \leq \sum_{n \in \mathscr{N}, i \in \mathscr{K}} p_{i, j, n} \leq p_{\max }, \quad \forall j \in \mathcal{S} \\
& \text { (13b) } \mathrm{R}_{i, f} \geq \gamma_{i}, \quad \forall i \in \mathscr{K} \\
& \text { (13c) } 0<\rho<1,
\end{array}
$$

where (13a) is the maximum transmit power constraint of SBSs, (13b) specifies minimum rate requirement, i.e., QoS of $\mathrm{UTs}$, and (13c) is the constraint of power splitting ratio. Since the formulated problem is not convex, to solve the established problem, we firstly convert nonconvex constraint (13b) into an equivalent form as follows:

$$
\begin{aligned}
\left(13 \mathrm{~b}^{\prime}\right) & : \rho \times p_{i, j, n} h_{i, j, n}^{2} \\
& -\left(\rho \sum_{j \notin \mathscr{B}_{i}} p_{i, j, n} h_{i, j, n}^{2}+\rho \sigma^{2}+\left(\sigma_{i}^{\mathrm{ID}}\right)^{2}\right) \\
& \times 2^{\left(\gamma_{i} / \mathrm{BW}_{i}-1\right)} \geq 0 .
\end{aligned}
$$

Then, problem (13) can be reformulated as follows:

$$
\begin{array}{ll}
\max _{(\rho, \mathbf{p})} & \sum_{i \in \mathscr{K}, f \in \mathscr{F}} \mathrm{EH}_{i, f} \\
\text { s.t. } & (13 \mathrm{a}),\left(13 \mathrm{~b}^{\prime}\right),(13 \mathrm{c}) .
\end{array}
$$

Since $\rho$ and $\mathbf{p}=\left(p_{i, j, n}\right)_{\mathrm{K} \times \mathrm{S} \times \mathrm{N}}$ are coupled in the objective of (15) and the condition $\left(13 b^{\prime}\right)$. Therefore, we propose an IPSPA algorithm to solve problem (15) by decoupling $\rho$ and $\mathbf{p}$. We fix $\rho$ firstly, and then (15) is converted from a nonconvex optimization problem into a convex one. Further, interior point method can be adopted to solve (15) [24]. Finally, the maximum harvested energy value can be obtained through alternately iterating $\rho$ and $\mathbf{p}$. The detail description of the proposed IPSPA algorithm to maximize the harvested energy of all UTs deployed in the SWIPT system with popularity cache scheme in dense small cell networks is shown in Algorithm 1. Energy harvesting coefficient, associated SBS, and subbandwidth for each UT are firstly determined in Algorithm 1. Then, optimal power allocation $\mathbf{p}^{*}$ and corresponding harvested energy for each $\rho$ are obtained by solving problem (15). Finally, the maximum value of harvested energy can be gained from all the cases of harvested energy.

\section{Complexity Analysis}

In Algorithm 1, the computational complexity of step (2) is $\mathcal{O}(\mathrm{FKS})$. Step (3) and step (4) have the computational complexity $\mathcal{O}\left(\left(\left(\rho_{\max }-\rho_{\text {min }}\right) \times \mathrm{V}_{\max }\right) / \rho_{\text {in }}\right)$, where $\mathcal{O}\left(\mathrm{V}_{\max }\right)$ is the complexity of interior point method. Both step (5) and step (8) have the computational complexity $\mathcal{O}(\mathrm{FK})$. Thus, the total computational complexity of Algorithm 1 is $\mathcal{O}\left(\left(\left(\rho_{\max }-\right.\right.\right.$ $\left.\left.\left.\rho_{\text {min }}\right) \times \mathrm{V}_{\text {max }} \times \mathrm{F}^{2} \mathrm{~K}^{2} \mathrm{~S}\right) / \rho_{\text {in }}+\mathrm{FK}\right) \approx \mathcal{O}\left(\left(\left(\rho_{\text {max }}-\rho_{\text {min }}\right) \times\right.\right.$ $\left.\left.V_{\max } \times \mathrm{F}^{2} \mathrm{~K}^{2} \mathrm{~S}\right) / \rho_{\text {in }}\right)$, which increases linearly with $\mathrm{F}^{2}, \mathrm{~K}^{2}$, and $\mathrm{S}$. Nevertheless, for the algorithm of joint user association, resource assignment, power splitting ratio, and power optimization given in [25], the computational complexity of user association and resource assignment is $\mathcal{O}\left(\mathrm{FS}^{\mathrm{K}}\right)$. Thus, the total computational complexity is $\mathcal{O}\left(\left(\left(\rho_{\max }-\rho_{\min }\right) \times\right.\right.$ $\left.\mathrm{V}_{\max } \times \mathrm{F}^{2} \mathrm{KS}^{\mathrm{K}}\right) / \rho_{\text {in }}$ ) when the algorithm runs to step (5), where the computational complexity of step (5) is $\mathcal{O}(\mathrm{FK})$. Moreover, the complexity of step (8) is $\mathcal{O}(\mathrm{FK})$. Therefore, the total complexity of joint user association, resource assignment, power splitting ratio, and power optimization is $\mathcal{O}\left(\left(\left(\rho_{\text {max }}-\rho_{\text {min }}\right) \times \mathrm{V}_{\text {max }} \times \mathrm{F}^{2} \mathrm{KS}^{\mathrm{K}}\right) / \rho_{\text {in }}+\mathrm{FK}\right) \approx \mathcal{O}\left(\left(\left(\rho_{\text {max }}-\right.\right.\right.$ $\left.\left.\left.\rho_{\text {min }}\right) \times \mathrm{V}_{\text {max }} \times \mathrm{F}^{2} \mathrm{KS}^{\mathrm{K}}\right) / \rho_{\text {in }}\right)$, which increases exponentially with the number of deployed UTs. Therefore, the proposed algorithm has a significantly reduced computational complexity.

\section{Simulation Results}

In our simulation, all the UTs and SBSs are randomly and uniformly distributed in the circular region with a radius of $20 \mathrm{~m}$. We consider the channel model with path loss, Rayleigh fading, and shadowing fading. The path loss from 
(1) Initialization $d=0, \mathcal{S}, \mathscr{F}, \mathscr{K}, \mathrm{C}, \rho_{\min }=0.001, \rho_{\max }=1$, and $\rho_{\text {in }}=0.001$.

(2) Calculate $\ell_{i, j, f}, \mathscr{B}_{i}$ and $\mathrm{BW}_{i}$.

(3) for $\rho=\rho_{\text {min }}: \rho_{\text {in }}: \rho_{\max }$ do

(4) Obtain $\mathbf{p} *$ through solving (15) by using interior point method given in [24].

(5) Substitute $\mathbf{p}^{d}=\mathbf{p}^{*}$ into the objective function of (15), and calculate corresponding harvested energy. $\mathrm{HE}\left(\mathbf{p}^{d}\right)=\sum_{i \in \mathscr{K}, f \in \mathscr{F}} \mathrm{EH}_{i, f}$

(6) Update $\rho^{d}=\rho, d=d+1$.

(7) end for

(8) Calculate the maximum harvested energy $\mathrm{HE}_{\text {max }}=\max \left(\mathrm{HE}\left(\mathbf{p}^{d}\right)\right)$.

(9) Output $\mathrm{HE}_{\text {max }}$, and corresponding $\mathbf{p}^{*}=\mathbf{p}^{l}$ and $\rho^{*}=\rho^{l}$.

Algorithm 1: IPSPA algorithm.
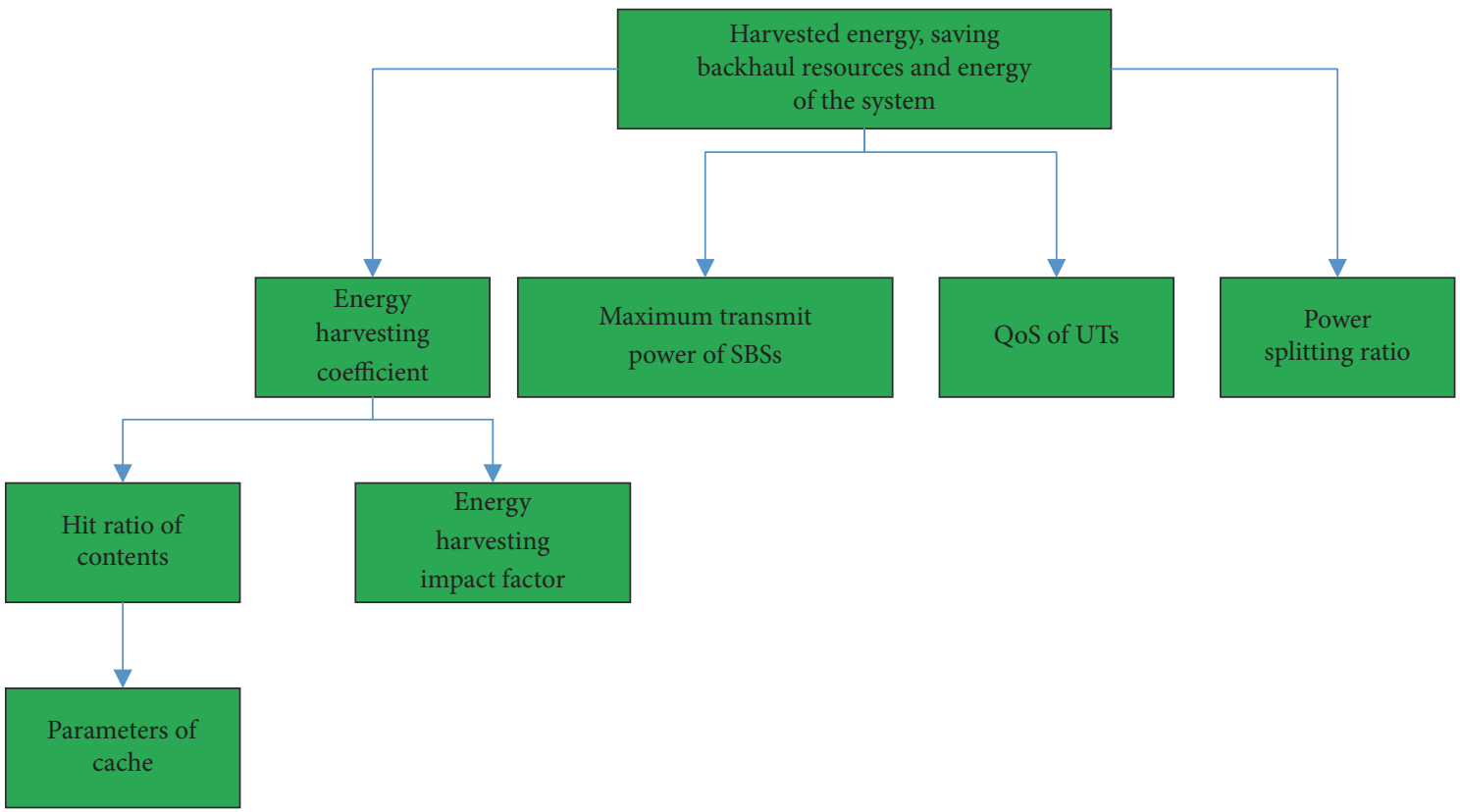

FIGURE 3: Relationship of different system parameters.

SBS $s$ to UT $u$ is set as $\mathrm{PL}_{s u}=38.46+20 * \log 10\left(d_{s u}\right)$, where $d_{s u}$ is the distance between SBS $s$ and UT $u$, with the unit $\mathrm{m}$. Besides, Rayleigh fading follows exponential distribution with parameter 1 . The standard deviation of the shadowing fading is $8 d B$ for the link between SBS and the UT [26]. Therefore, $h(i, j, n)=\operatorname{Rayleigh}(i, j, n) *$ $10^{\left(\left(-P L_{s u}(i, j)-\text { shadowing }(i, j, n)\right) / 10-3\right)}$ denotes the channel gain from SBS $j$ to UT $i$ on subband $n$. Besides, we set $\sigma^{2}=-50 d B m$ and $\left(\sigma_{i}^{\mathrm{ID}}\right)^{2}=-30 \mathrm{dBm}$, respectively. Assume that each UT randomly requests a content in $1 \mathrm{~s}$, and the size of each content is 20 kbit. Moreover, we set $\eta_{B H}=0.68$, i.e., $m=1.47, p_{\max }=$ $1 \mathrm{w}$, and the number of OFDMA RBs as 50 , respectively.

Our proposed SWIPT system with popularity cache scheme has the advantages of improving harvested energy of UTs and saving backhaul resources and energy. The performance of the system is influenced by different system parameters given in Figure 3. Therefore, we carry out the simulation from the aspects given in Figures 4, 5, 6, 7, 8, 9, 10, and 11. Additionally, we compare our proposed scheme with the following schemes.

(i) Uniform cache scheme is given in $[18,19]$ applied in our proposed SWIPT system.

(ii) No caching scheme (nocache) of SWIPT system is given in [6].

Firstly, we simulate the hit ratio of our proposed popularity cache scheme and uniform cache scheme given in $[18,19]$.

Figure 4 shows the hit ratio with respect to the content index. We set the total number of contents as $10, \alpha=0.5$ for blue and black curves, and $C=6$ for green and red curves. We can observe that for a content under the same popularity distribution parameter $\alpha$, hit ratio of the content increases with cache size. Moreover, for a content under the same cache size of SBSs, hit ratio of the content becomes larger when popularity distribution parameter $\alpha$ increases. Besides, hit ratio for a content with high popularity of our 


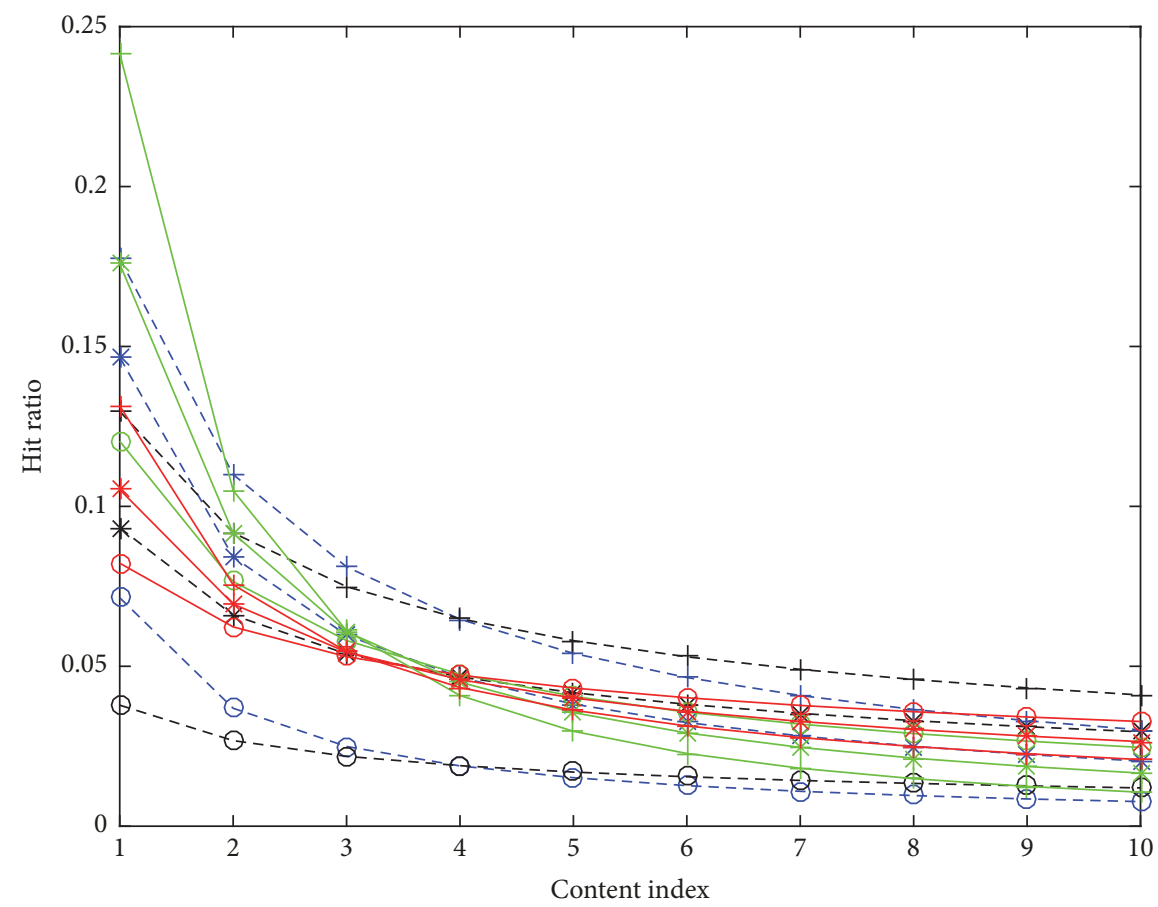

$$
\begin{aligned}
& \text { - } \ominus-\text {. popularity-cache }=2 \\
& \text { - popularity-alfa }=0.4 \\
& \text { - } \odot \text {-. uniform-cache }=2 \\
& \bigcirc \text { uniform-alfa }=0.4 \\
& \text { - - * - popularity-cache }=6 \\
& \text { * popularity-alfa }=0.6 \\
& \text { - * - uniform-cache }=6 \\
& \text { * uniform-alfa }=0.6 \\
& -+ \text { popularity-cache }=10 \\
& + \text { popularity-alfa }=0.8 \\
& -+{ }_{-} \text {. uniform-cache }=10 \\
& \text { । uniform-alfa }=0.8
\end{aligned}
$$

Figure 4: Hit ratio varies with content index.

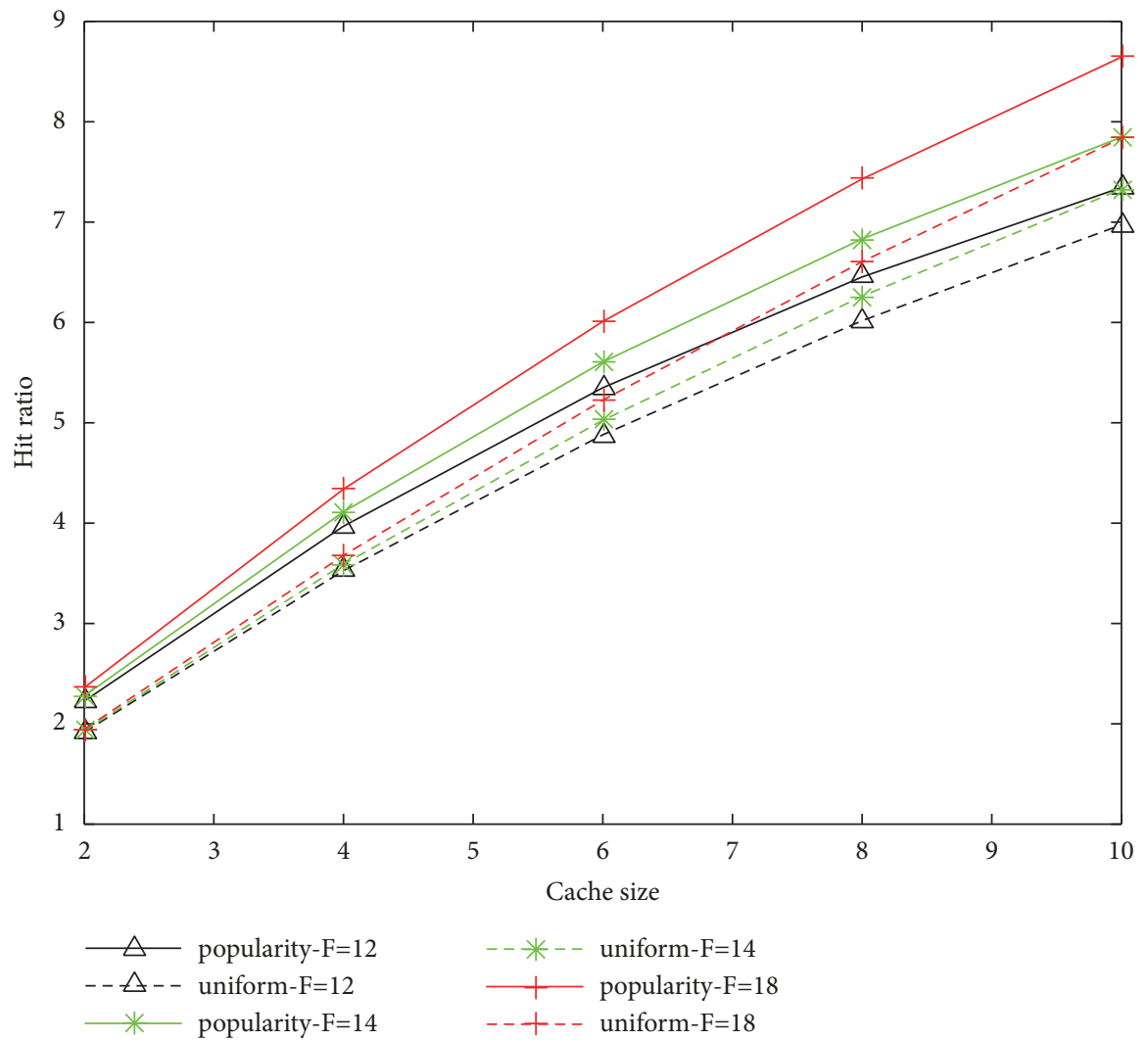

FIgURE 5: Hit ratio varies with cache size $C$. 


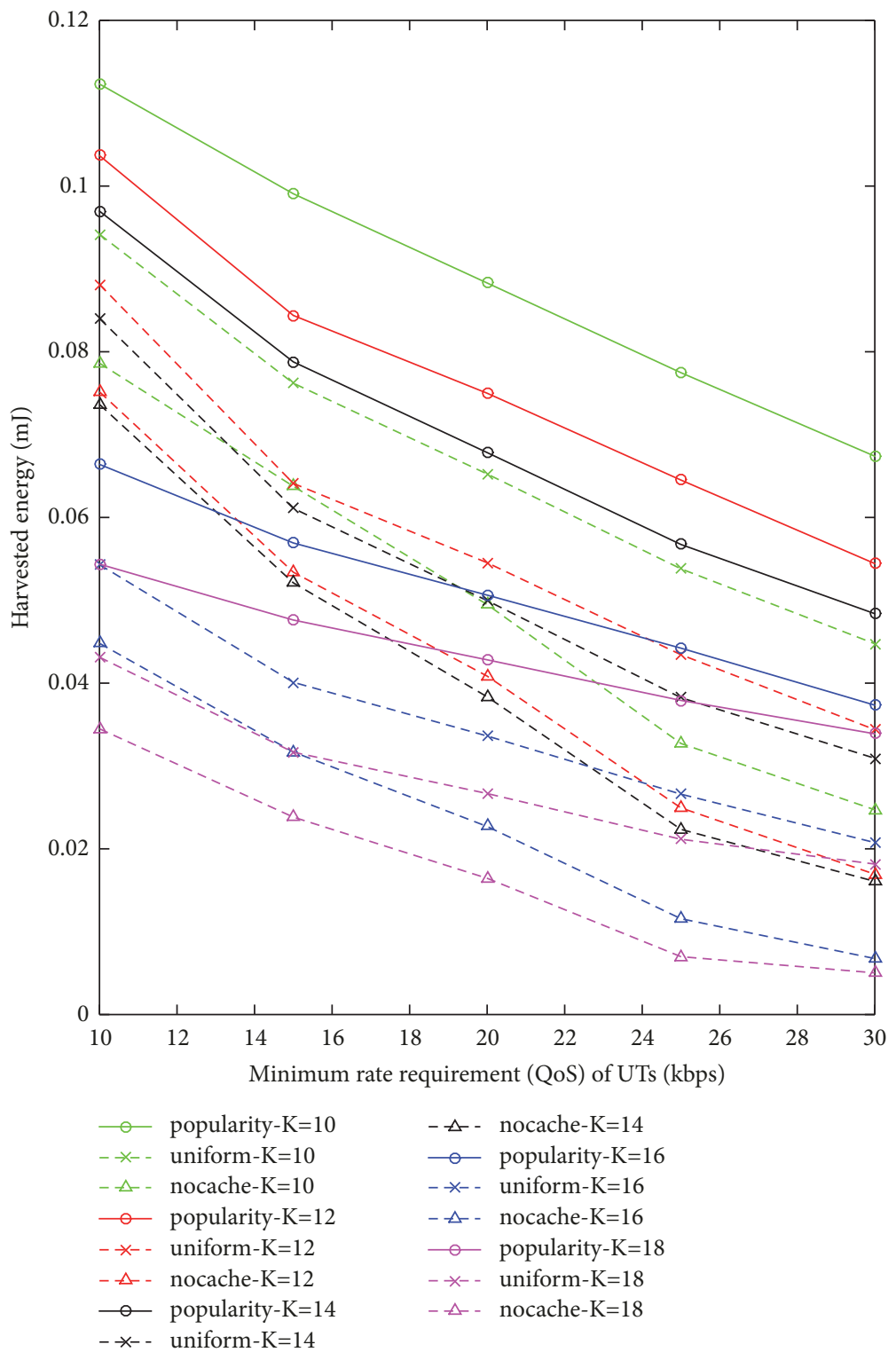

FIGURE 6: Harvested energy varies with QoS of UTs under different K.

proposed popularity cache scheme is larger than uniform cache scheme when cache size of SBSs and popularity distribution parameter $\alpha$ are the same.

Figure 5 shows hit ratio with respect to C. We set $\alpha=0.5$. We can observe that our proposed popularity cache scheme has better hit ratio than uniform scheme. Moreover, the hit ratio of our proposed and uniform schemes increases with the increasing of $\mathrm{C}$ under the same number of contents $\mathrm{F}$. The reason is that the probability of contents being cached increases with the increasing of $\mathrm{C}$. Additionally, the hit ratio of our proposed and uniform schemes increases with the total number of contents under the same $\mathrm{C}$. Then, we compare the energy harvesting property of our proposed popularity cache scheme with the uniform cache scheme given in $[18,19]$ and the no caching scheme (nocache) given in [6].

Figure 6 shows that harvested energy of the system with respect to minimum rate requirement, i.e., QoS of UTs under different number of UTs $K$, where $C=6$ and $S=8$. We can observe that harvested energy decreases with the number of deployed UTs K. The reason is that more power is used to provide service for UTs. Moreover, we can see that our proposed scheme has better performance than uniform cache scheme and no caching scheme. The reason is that our proposed scheme has larger energy harvesting coefficients through improving the probability of obtaining contents from local SBSs.

Figure 7 shows that harvested energy of the system varies with the minimum rate requirement, i.e., QoS of UTs under different $C$, where $K=14$ and $S=8$. We can intuitively observe that harvested energy increases with $C$. The reason is that energy harvesting ability of our proposed scheme increases with C. Besides, we can see that our proposed popularity cache scheme has better performance than the uniform cache scheme and the no caching scheme because the proposed 


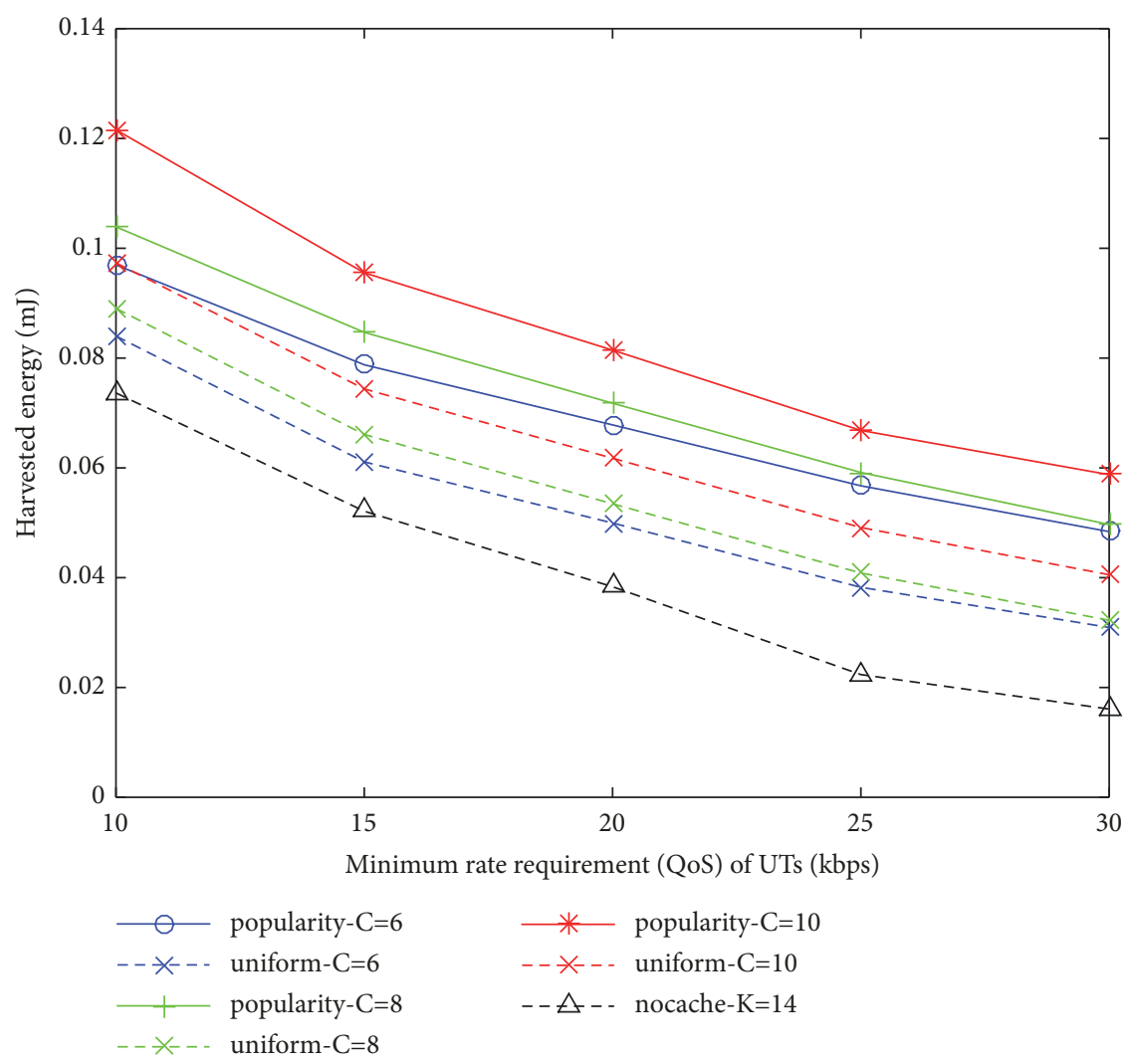

FIgURE 7: Harvested energy varies with QoS of UTs under different C.

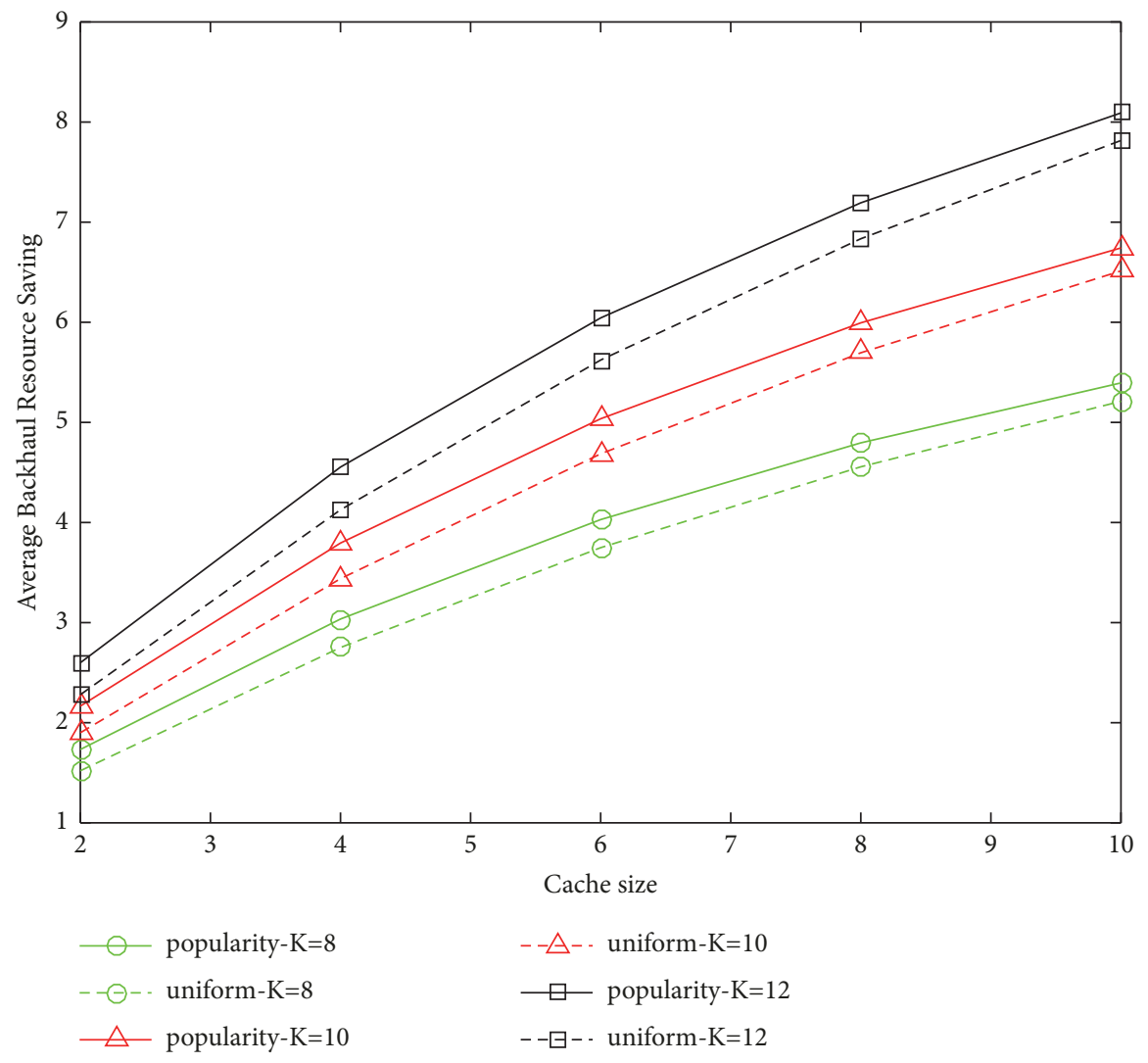

FIGURE 8: Average backhaul resource saving varies with cache size. 


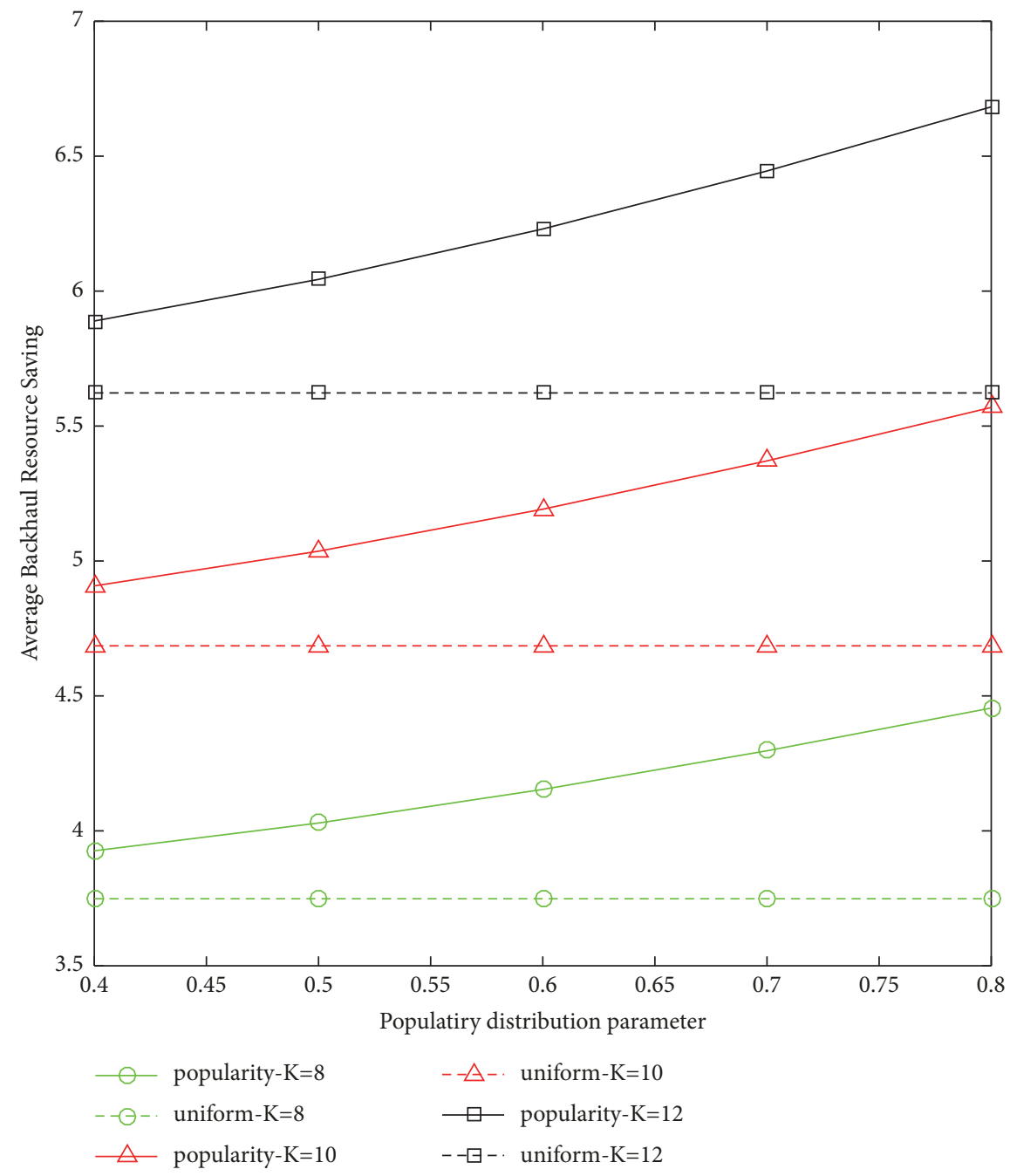

FIGURE 9: Average backhaul resource saving varies with popularity distribution parameter $\alpha$.

scheme can achieve higher hit ratio for the content with high popularity.

Finally, we compare our proposed cache scheme with the uniform cache scheme given in $[18,19]$ in terms of the ability of saving backhaul resources and energy. Average saving backhaul resource is depicted in Figures 8 and 9. We assume that each content consumes 1 unit backhaul resource when it is fetched through the backhaul link.

Figure 8 shows the average backhaul resource saving with respect to the size of cache C. We can observe that our proposed popularity cache scheme has better performance than uniform cache scheme. Moreover, our proposed popularity cache scheme can save more backhaul resources when cache size $C$ becomes larger. Additionally, our proposed popularity cache scheme has better performance than uniform cache scheme when the number of deployed UTs K in dense small cell networks increases. The reason is that our proposed scheme has higher hit ratio and energy harvesting coefficient.

Figure 9 shows the average backhaul resource saving with respect to popularity distribution parameter $\alpha$. We can observe that the performance of uniform cache scheme is not influenced by the parameter $\alpha$, while our proposed popularity cache scheme can save more backhaul resources with the increasing of parameter $\alpha$. Moreover, our proposed popularity cache scheme has better performance than uniform cache scheme as $\alpha$ increases. The reason is that our proposed scheme guarantees more contents obtained from local SBSs. As given in $[27,28]$, the average saving energy of backhaul link can be calculated as follows:

$$
\begin{aligned}
P_{\mathrm{BH}}^{\text {save }} & =\overline{p_{h i t}} \times\left(\frac{\mathrm{S}}{\max _{d l}} \times P_{s}+\mathrm{S} \times P_{d l}\right) \\
& =\sum_{j \in \mathcal{S}, f \in \mathscr{F}} \mathrm{A}_{i, j, f}^{h i t} \times \mathrm{K} \times\left(\frac{\mathrm{S}}{\max _{d l}} \times P_{s}+\mathrm{S} \times P_{d l}\right)
\end{aligned}
$$

where $\overline{p_{h i t}}$ is the average hit probability of all contents when the number of UTs is $\mathrm{K}, \max _{d l}$ is the maximum number of downlink available aggregation switch interfaces, and $P_{d l}$ is the power consumption of one downlink interface in the aggregation switch for receiving the backhauled traffic. Moreover, $P_{s}=\delta \times P_{\max }+(1-\delta) \times U_{\text {total }} / U_{\max } \times P_{\max }$ denotes 


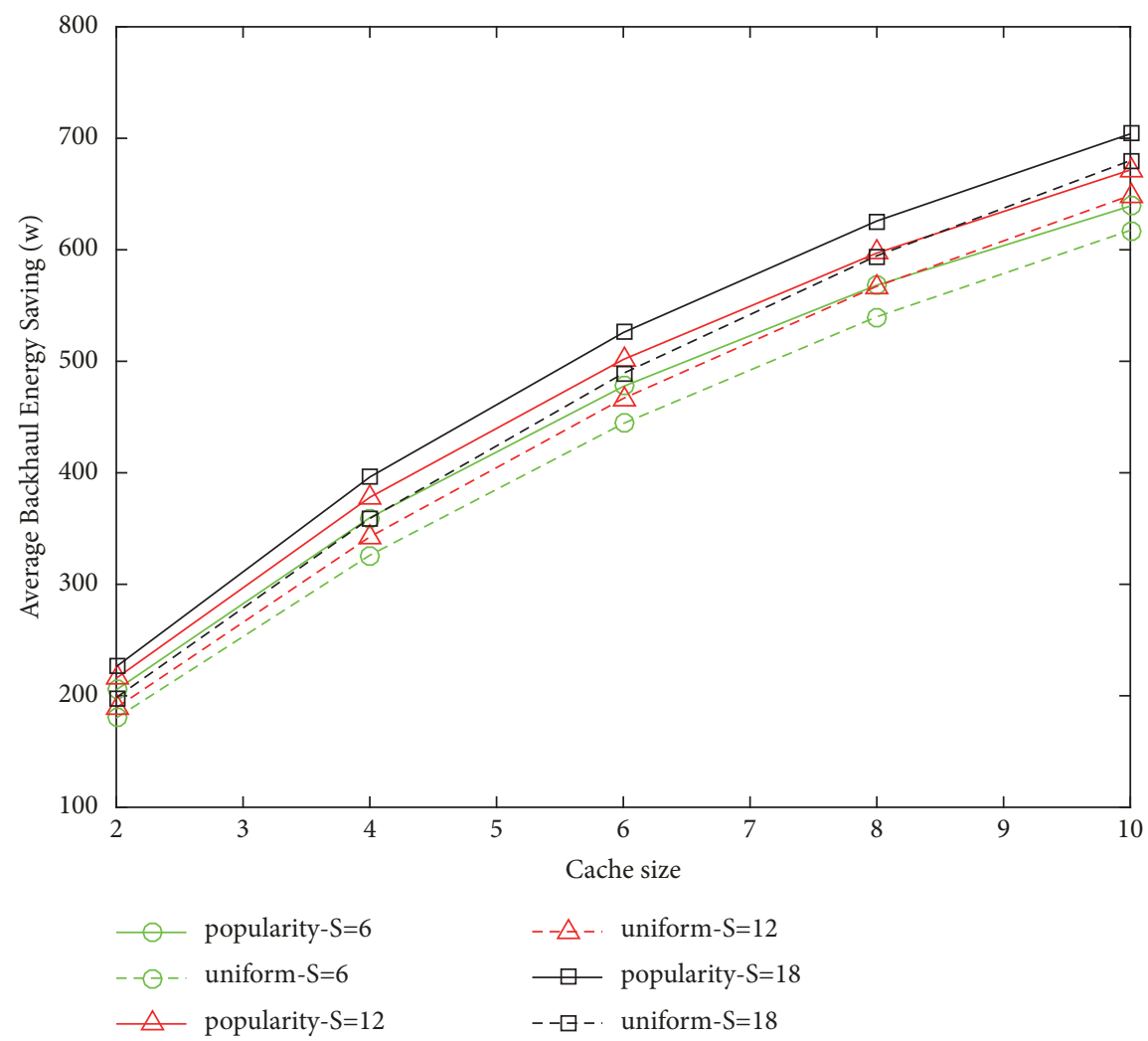

FIGURE 10: Average backhaul energy saving varies with cache size.

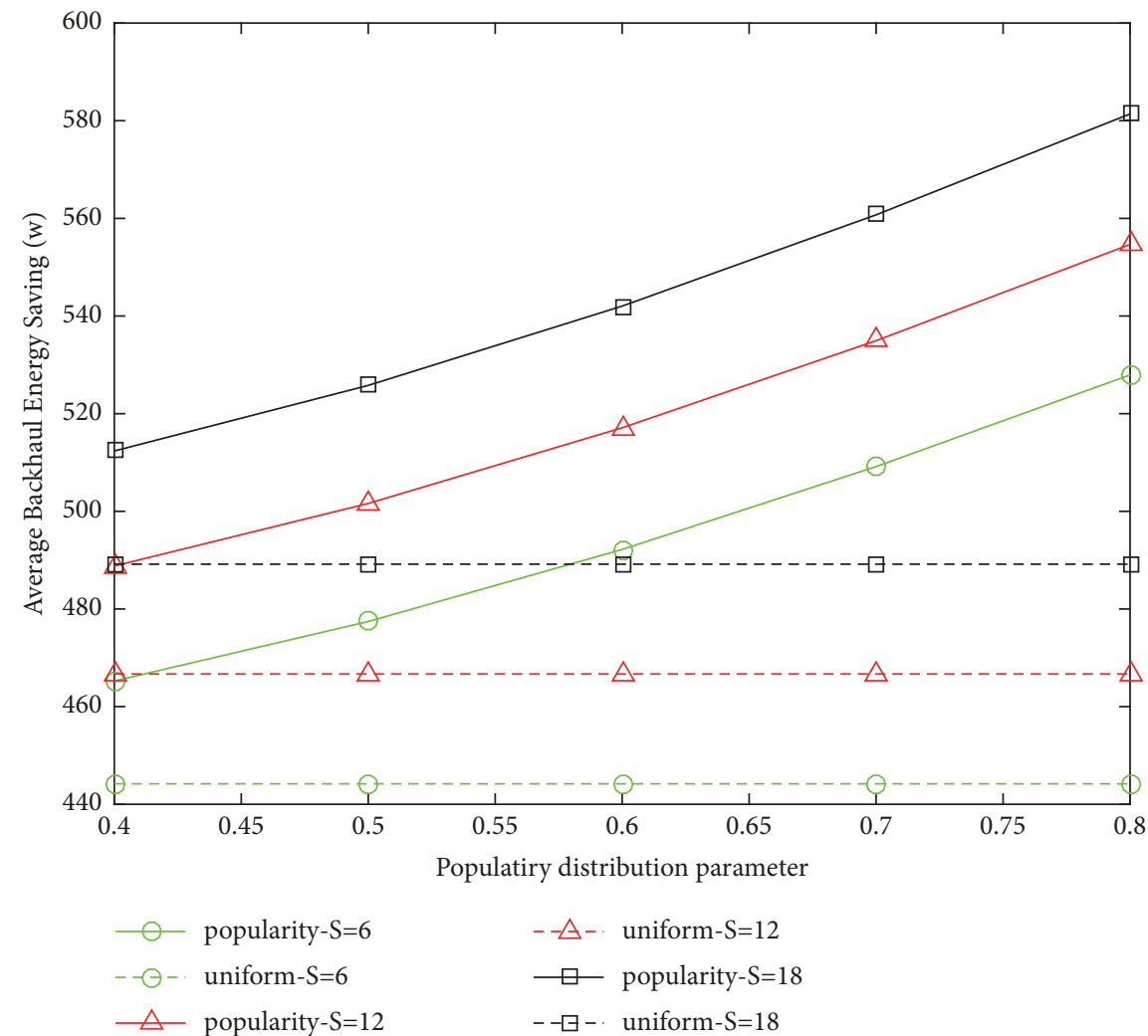

FIGURE 11: Average backhaul energy saving varies with popularity distribution parameter $\alpha$. 
the power consumed by a switch, where $P_{\max }$ represents the maximum power consumption of the switch, $U_{\max }$ is the maximum amount of traffic a switch can deal with, and $U_{\text {total }}$ represents the amount of traffic that is traversing the switch that is influenced by the parameter $\delta \in(0,1)$. We set $\mathrm{K}=8, \mathrm{~F}=10, \max _{d l}=24, p_{d l}=1 \mathrm{w}, \delta=0.9, P_{\max }=$ $300 \mathrm{w}, U_{\max }=36 \mathrm{Gbps}$, and $\gamma_{i}=20 \mathrm{kbps}$.

Figure 10 shows average backhaul saving energy with respect to the cache size $\mathrm{C}$, where $\alpha=0.5$. We can see that our proposed popularity cache scheme has better performance than uniform cache scheme. Moreover, our proposed popularity cache scheme can save more backhaul resources when cache size $\mathrm{C}$ becomes larger. The reason is that hit ratio of our proposed scheme increases with the increasing of cache size $\mathrm{C}$, which improves energy harvesting coefficient. Besides, our proposed scheme has better performance than uniform cache scheme with the increasing number of deployed SBSs S since it ensures more UTs get requested contents from local SBSs.

Figure 11 illustrates average backhaul saving energy with respect to popularity distribution parameter $\alpha$, where $\mathrm{C}=6$. We can observe that the performance of uniform cache scheme is not affected by the parameter $\alpha$. However, our proposed popularity cache scheme can save more backhaul resources when $\alpha$ increases. The reason is that hit ratio and energy harvesting coefficient increase with the increasing of $\alpha$. Moreover, our proposed popularity cache scheme has better performance than uniform cache scheme with the increasing of parameters $\alpha$ and $S$ because it ensures more contents requested by UTs obtained through local SBSs without adding burden on backhaul link.

\section{Conclusion}

In this paper, the harvested energy maximization problem of SWIPT system with popularity cache scheme in dense small cell networks has been studied. Firstly, system model consisting of the network model, content request, and popularity cache scheme was provided. Then, a harvested energy maximization problem of SWIPT system with popularity cache scheme was established. Further, an IPSPA algorithm was given to solve the formulated problem. Finally, we demonstrated that the proposed SWIPT system with popularity cache scheme can improve harvested energy of UTs and save backhaul resources and energy through a number of simulations.

\section{Data Availability}

The data used to support the findings of this study are included within the article.

\section{Disclosure}

Jiandong Li serves as a Senior Member of IEEE.

\section{Conflicts of Interest}

The authors declare that there are no conflicts of interest regarding the publication of this paper.

\section{Acknowledgments}

This paper is supported in part by the National Natural Science Foundation of China [nos. 91638202, 61231008, and 61571351].

\section{References}

[1] J. Huang, C. Xing, and C. Wang, "Simultaneous wireless information and power transfer: technologies, applications, and research challenges," IEEE Communications Magazine, vol. 55, no. 11 , pp. 26-32, 2017.

[2] F. Jameel, M. A. A. Haider, A. A. Butt et al., "A technical review of simultaneous wireless information and power transfer (SWIPT)," in Proceedings of the IEEE International Symposium on Recent Advances in Electrical Engineering, pp. 1-6, 2017.

[3] T. D. P. Perera, D. N. K. Jayakody, S. K. Sharma, S. Chatzinotas, and J. Li, "Simultaneous wireless information and power transfer (SWIPT): recent advances and future challenges," IEEE Communications Surveys and Tutorials, vol. 20, no. 1, pp. 264302, 2018.

[4] J. Ding, L. Jiang, and C. He, "User-centric energy-efficient resource management for time switching wireless powered communications," IEEE Communications Letters, vol. 22, no. 1, pp. 165-168, 2018.

[5] D. Mishra and G. C. Alexandropoulos, "Jointly optimal spatial channel assignment and power allocation for MIMO SWIPT systems," IEEE Wireless Communications Letters, vol. 7, no. 2, pp. 214-217, 2018.

[6] Z. Hu, N. Wei, and Z. Zhang, "Optimal resource allocation for harvested energy maximization in wideband cognitive radio network with SWIPT," IEEE Access, vol. 5, pp. 23 383-23 394, 2017.

[7] N. Janatian, I. Stupia, and L. Vandendorpe, "Optimal resource allocation in ultra-low power fog-computing SWIPT-based networks," in Proceedings of the IEEE Wireless Communications and Networking Conference, pp. 1-6, IEEE, 2018.

[8] H. Chai, S. Leng, J. Hu, and K. Yang, "Resources allocation in SWIPT aided fog computing networks," in Proceedings of the 9th International Conference on Advanced Infocomm Technology, ICAIT '17, pp. 239-244, November 2017.

[9] Y. Ye, Y. Li, D. Wang, F. Zhou, R. Q. Hu, and H. Zhang, "Optimal transmission schemes for df relaying networks using SWIPT," IEEE Transactions on Vehicular Technology, vol. 67, no. 8, pp. 7062-7072, 2018.

[10] Y. Dong, M. J. Hossain, and J. Cheng, "Joint power control and time switching for SWIPT systems with heterogeneous QoS requirements," IEEE Communications Letters, vol. 20, no. 2, pp. 328-331, 2016.

[11] G. Pan, H. Lei, Y. Yuan, and Z. Ding, "Performance analysis and optimization for SWIPT wireless sensor networks," IEEE Transactions on Communications, vol. 65, no. 5, pp. 2291-2302, 2017.

[12] D. W. K. Ng and R. Schober, "Resource allocation for coordinated multipoint networks with wireless information and power transfer," in Proceedings of the Global Communications 
Conference, GLOBECOM '14, pp. 4281-4287, IEEE, December 2014.

[13] L. Xu, J. Wang, H. Zhang, and T. A. Gulliver, "Performance analysis of IAF relaying mobile D2D cooperative networks," Journal of The Franklin Institute, vol. 354, no. 2, pp. 902-916, 2017.

[14] L. Xu, J. Wang, Y. Liu, J. Yang, W. Shi, and T. A. Gulliver, "Outage performance for IDF relaying mobile cooperative networks," in Proceedings of the International Conference on $5 G$ for Future Wireless Networks, pp. 395-402, 2017.

[15] A. Liu and V. K. N. Lau, "Capacity gain of physical layer caching in backhaul-limited dense wireless networks," in Proceedings of the Computer Communications Conference, pp. 64-72, IEEE, April 2015.

[16] J. Song and W. Choi, "Minimum cache size and backhaul capacity for cache-enabled small cell networks," IEEE Wireless Communications Letters, vol. 7, no. 4, pp. 490-493, 2018.

[17] R. Hou, Y. Cheng, L. X. Cai, and H. Zhuang, "Performance evaluation for caching-based content distribution in backhaullimited small cell networks," in Proceedings of the International Conference on Wireless Communications and Signal Processing (WCSP '15), pp. 1-5, October 2015.

[18] Y. Wang, X. Tao, X. Zhang, and Y. Gu, "Cooperative caching placement in cache-enabled D2D underlaid cellular network," IEEE Communications Letters, vol. 21, no. 5, pp. 1151-1154, 2017.

[19] J. Li, Y. Chen, M. Ding, F. Shu, B. Vucetic, and X. You, "A smallcell caching system in mobile cellular networks with LoS and NLoS channels," IEEE Access, vol. 5, pp. 1296-1305, 2017.

[20] F. Cheng, Y. Yu, Z. Zhao, N. Zhao, Y. Chen, and H. Lin, "Power allocation for cache-aided small-cell networks with limited backhaul," IEEE Access, vol. 5, pp. 1272-1283, 2017.

[21] A. Kumar and W. Saad, "On the tradeoff between energy harvesting and caching in wireless networks," in Proceedings of the IEEE International Conference on Communication Workshop, ICCW' '15, pp. 1976-1981, June 2015.

[22] X. Peng, J. Shen, J. Zhang, and K. B. Letaief, "Backhaul-aware caching placement for wireless networks," in Proceedings of the Global Communications Conference, pp. 1-6, IEEE, December 2015.

[23] H. Ahlehagh and S. Dey, "Video-aware scheduling and caching in the radio access network," IEEE/ACM Transactions on Networking, vol. 22, no. 5, pp. 1444-1462, 2014.

[24] S. Boyd and L. Vandenberghe, Convex Optimization, Cambridge University Press, 2004.

[25] M. Ali, Q. Rabbani, M. Naeem, S. Qaisar, and F. Qamar, "Joint user association, power allocation, and throughput maximization in 5G H-CRAN networks," IEEE Transactions on Vehicular Technology, vol. 66, no. 10, pp. 9254-9262, 2017.

[26] E. U. T. R. Access, "Further advancements for E-UTRA physical layer aspects," Tech. Rep. 3GPP TR 36.814, 2010.

[27] S. Tombaz, P. Monti, K. Wang, A. Västberg, M. Forzati, and J. Zander, "Impact of backhauling power consumption on the deployment of heterogeneous mobile networks," in Proceedings of the Global Telecommunications Conference, pp. 1-5, IEEE, December 2011.

[28] K. M. S. Huq, S. Mumtaz, J. Bachmatiuk, J. Rodriguez, X. Wang, and R. L. Aguiar, "Green HetNet CoMP: energy efficiency analysis and optimization," IEEE Transactions on Vehicular Technology, vol. 64, no. 10, pp. 4670-4683, 2015. 


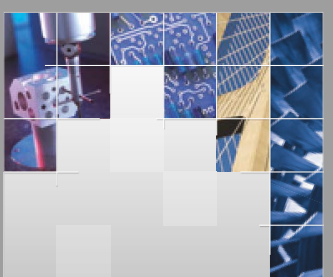

\section{Enfincering}
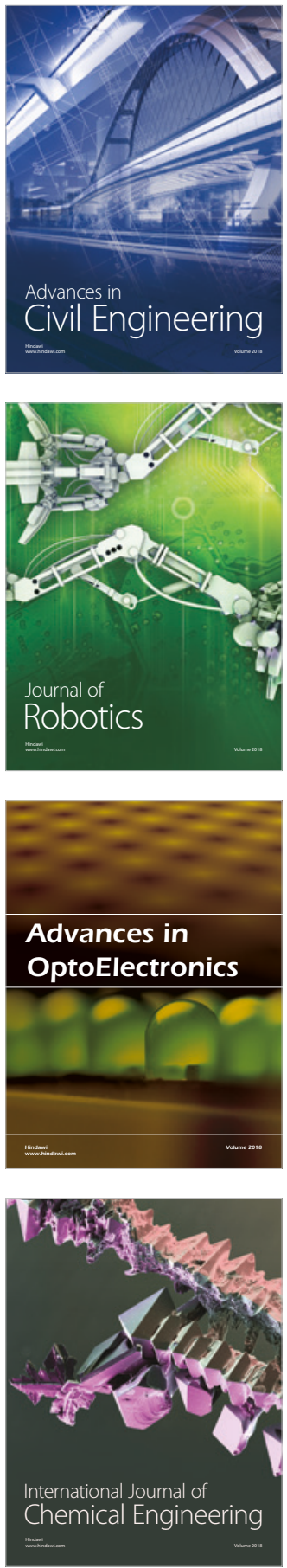

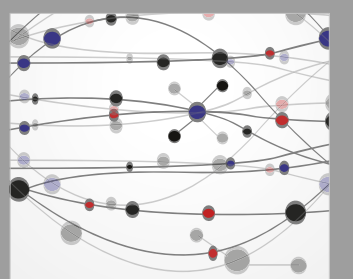

\section{Rotating \\ Machinery}

The Scientific World Journal

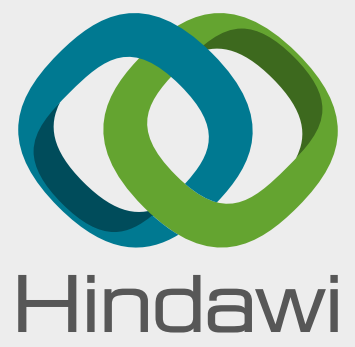

Submit your manuscripts at

www.hindawi.com
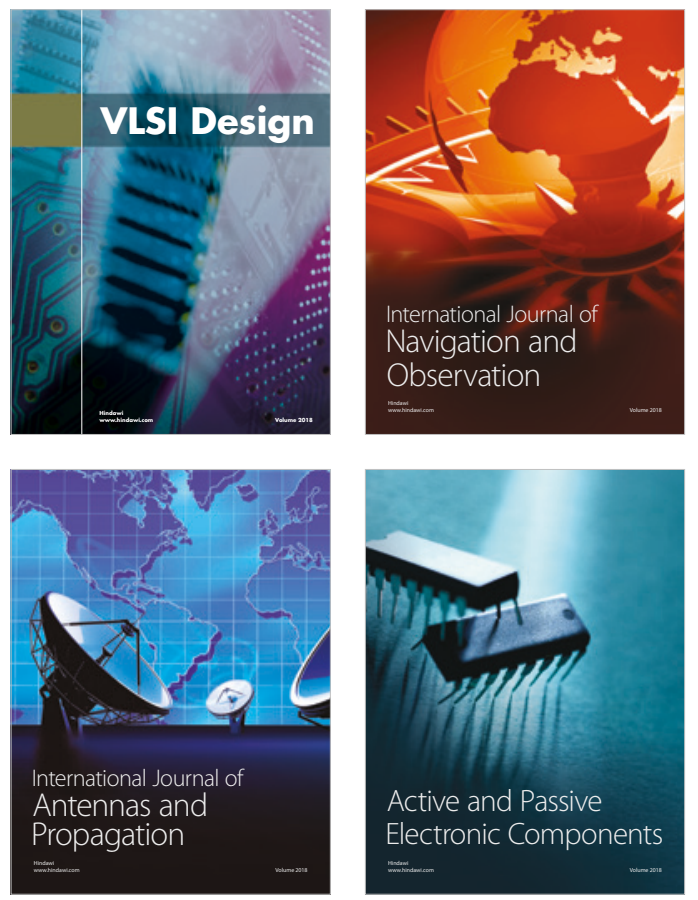
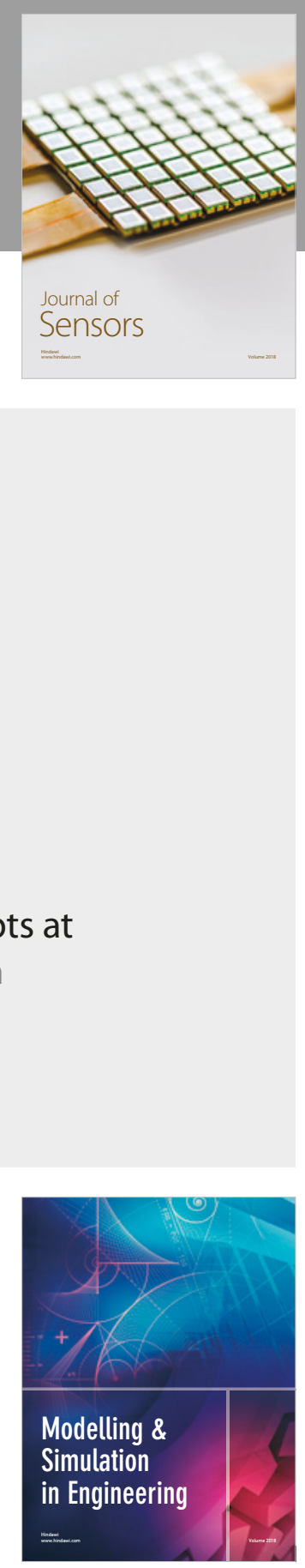

\section{Advances \\ Multimedia}
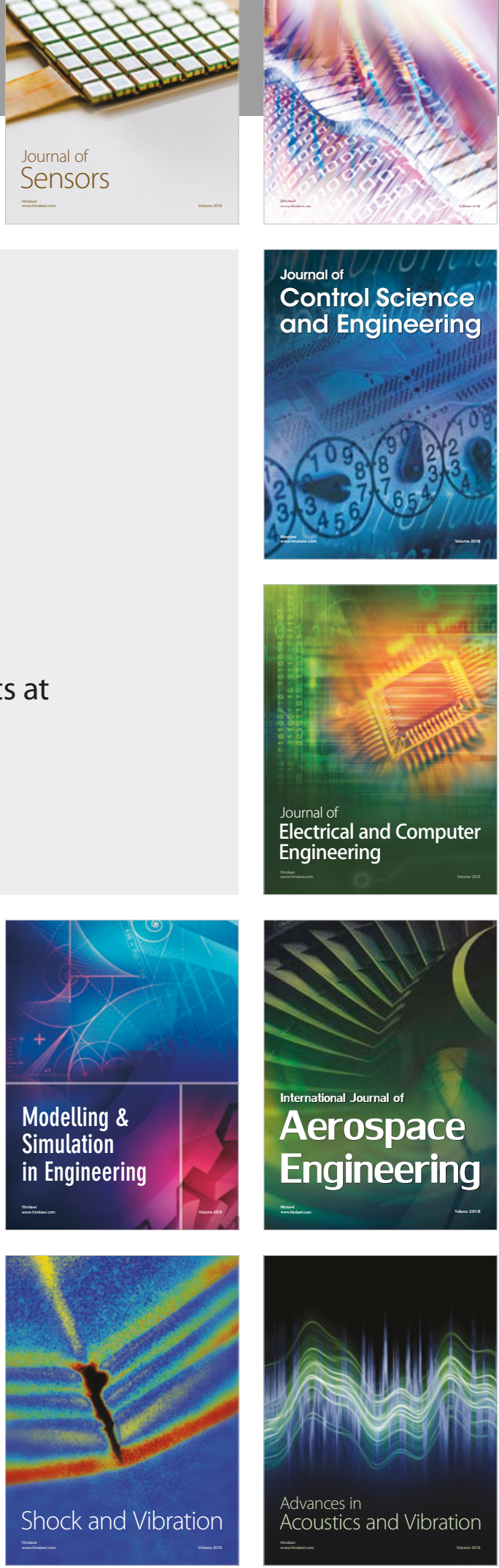